\section{Rec $\mathrm{C}_{\mathrm{A}} \ldots$}

Revista Colombiana de Ciencia Animal ISSN: 2027-4297

reciaeditor@unisucre.edu

Universidad de Sucre

Colombia

\title{
Perfil hematológico durante la gestación de Ovinos de Pelo Criollos en el departamento de Córdoba, Colombia
}

Plaza Cuadrado, Alberto; Hernández Padilla, Eduardo; Rugeles Pinto, Clara; Vergara Garay, Oscar; Herrera Benavides, Yonairo

Perfil hematológico durante la gestación de Ovinos de Pelo Criollos en el departamento de Córdoba, Colombia

Revista Colombiana de Ciencia Animal, vol. 11, núm. 1, 2019

Universidad de Sucre, Colombia

DOI: https://doi.org/10.24188/recia.v0.n0.2019.657

Los autores permiten a RECIA reimprimir el material publicado en él. En caso de que un autor quiera traducir o usar una publicación parcial o completa de nuestro Diario, el autor debe obtener un permiso por escrito del editor de la revista.

Esta obra está bajo una Licencia Creative Commons Atribución 4.0 Internacional. 


\section{Perfil hematológico durante la gestación de Ovinos de Pelo Criollos en el departamento de Córdoba, Colombia}

Hematological profile during the gestation of Creole Hair Sheep in the department of Córdoba, Colombia

Alberto Plaza Cuadrado

DOI: https://doi.org/10.24188/recia.v0.n0.2019.657

Trabajo independiente, Colombia

alsepla2014@gmail.com

(iD http://orcid.org/0000-0003-0422-8267

Recepción: 02 Octubre 2018

Eduardo Hernández Padilla

Aprobación: 15 Enero 2019

Trabajo independiente, Colombia

Publicación: 05 Febrero 2019

eduardinhnz95@gmail.com

(D) http://orcid.org/0000-0002-9746-835X

Clara Rugeles Pinto

Universidad de Córdoba, Departamento de Ciencias

Pecuarias, Grupo de Investigación en Producción Animal

Tropical - GIPAT, Montería, Colombia

crugeles@correo.unicordoba.edu.co

(iD) http://orcid.org/0000-0002-5610-5712

Oscar Vergara Garay

Universidad de Córdoba, Departamento de Ciencias

Pecuarias, Grupo de Investigación en Producción Animal

Tropical - GIPAT, Montería, Colombia

overgara@correo.unicordoba.edu.co

(D) http://orcid.org/0000-0002-1027-9469

Yonairo Herrera Benavides

Universidad de Córdoba, Departamento de Ciencias

Pecuarias, Grupo de Investigación en Producción Animal

Tropical - GIPAT, Montería, Colombia

yonairoherrerab@unicordoba.edu.co

(iD) http://orcid.org/0000-0002-8586-7150

\section{RESUMEN:}

Objetivo. Determinar valores hematológicos en Ovinos Criollos de Pelo hembras durante la fase de gestación en el departamento de Córdoba. Materiales y métodos. Se analizaron 74 muestras de sangre extraídas de la vena yugular en tubos BD Vacutainer al vacío con anticoagulante EDTA de ovejas gestantes divididas en 4 grupos ( $<31,31$ a 60,61 a 90 y $>91$ días de gestación) a las que se les realizaron hemogramas con un analizador automático. Resultados. Los resultados de la presente investigación para las variables hematológicas de ovejas criollas colombianas no presentaron diferencias entre los grupos de gestación, en su mayoría, concuerdan con los reportes de otras investigaciones realizadas en razas criollas tanto de ovejas como de cabras de otros países. Los resultados muestran diferencia significativa $(\mathrm{p} \leq 0.05)$ en el porcentaje de monocitos con relación al periodo de gestación en ovejas criollas. Conclusiones. La presente investigación podría establecer la base para la interpretación clínica y determinar durante la gestación en la oveja, si el porcentaje de monocitos corresponde a los valores normales.

\section{NotAS DE AUTOR}


Palabras Clave: Ovis aries, ovejas, hemograma, preñez.

\section{Abstract:}

Objective. To determine haematological values in Female Hair Creole Ovines during the gestation phase in the department of Córdoba. Materials and methods. We analyzed 74 samples of blood extracted from the jugular vein in vacuum BD Vacutainer ${ }^{\circ}$ tubes with EDTA anticoagulant of pregnant sheep divided into 4 groups ( $<31,31$ to 60,61 to 90 and $>91$ days of gestation) to which were made blood tests with an automatic analyzer. Results. The results of the present investigation for the hematological variables of Colombian Creole sheep did not show differences between the gestation groups, in their majority, they agree with the reports of other investigations carried out in Creole breeds of sheep and goats from other countries. The results show a significant difference $(p \leq 0.05)$ in the percentage of monocytes in relation to the gestation period in Creole sheep. Conclusions. The present investigation could establish the basis for clinical interpretation and determine during pregnancy in sheep, if the percentage of monocytes corresponds to normal values.

KEYwORDS: Ovis aries, sheep, blood count, pregnancy.

\section{INTRODUCCIÓN}

Los ovinos de Colombia tienen su origen en la conquista, los españoles introdujeron los primeros ejemplares de lana y pelo; los primeros provenientes de Europa y los segundos del África occidental. Después de 500 años vemos como los ovinos aún están por desarrollar su potencialidad (1).

La producción ovina en Colombia se ha incrementado en los últimos años e impactado en la economía agropecuaria, debido posiblemente a las bondades de dicha producción, que la hacen diferente a las demás y le dan un sello característico (2).

Reportes de investigación científica demuestran en sus resultados ciertas diferencias en valores hematológicos relacionados con los factores de variabilidad, entre los más estudiados se encuentran los diferentes estados fisiológicos y las razas (3).

Las variaciones en el estado fisiológico de los animales repercuten sobre variables hematológicas. La gestación, periodo de lactancia, edad y sexo han sido mencionados en distintas especies animales (bovinos, ovinos, caprinos, entre otras) como causantes de variaciones en los valores hematológicos normales. Por esta razón, para una correcta interpretación del hemograma, es necesario considerar la influencia de dichos factores de variabilidad, al igual que las condiciones climáticas y ambientales, estado nutricional, raza y manejo zootécnico (3).

La mayoría de los padecimientos son reflejados en la sangre, por lo tanto, es necesario realizar hematología, ya que permite evaluar las líneas celulares de la sangre y así caracterizar el comportamiento de éstas durante la gestación. Cabe destacar, que la hematología es una herramienta de diagnóstico clínico que permite conocer el estado de distintos órganos (4). Pero, para que esta herramienta diagnóstica sea utilizable y arroje información relevante, es necesario que se conozcan los valores normales en ovejas gestantes Criollas de Pelo colombianas, con el fin de comparar valores normales y anormales, generar rangos confiables y efectuar las correcciones pertinentes en esta área de la producción. Por esta razón, el objetivo del presente estudio fue determinar valores hematológicos en ovejas Criollas de Pelo colombianas durante la fase de gestación en el departamento de Córdoba.

\section{MATERIALES Y MÉTODOS}

Tipo de estudio. Se realizó un estudio descriptivo de corte transversal con 74 ovejas en periodo de gestación tomadas a conveniencia según disponibilidad en cada predio desde agosto a diciembre de 2016.

Localización. El estudio se realizó en granjas del departamento de Córdoba, Colombia. Ubicado entre las coordenadas $7^{\circ} 23^{\prime}$ y $9^{\circ} 26^{\prime}$ de latitud Norte y los $74^{\circ} 52^{\prime}$ y $76^{\circ} 32^{\prime}$ de longitud Oeste, a una altitud promedio 
de $30 \mathrm{~m}$, temperatura promedio anual de $28^{\circ} \mathrm{C}$, humedad relativa del $84 \%$, precipitación anual de $1200 \mathrm{~mm}$ y pertenece a la formación climática de bosque tropical lluvioso (5).

Animales de estudio. Se utilizaron ovejas clínicamente sanas, mayores de dos años en periodo de gestación con peso de 30-35 kg. Se determinó tiempo de preñez por medio de registros de monta. El número de animales evaluados estuvo dado por la disponibilidad de hembras gestantes en cada finca y se realizó un único muestreo sanguíneo en época de lluvia. Con el fin de hacer una evaluación más detallada de toda la gestación se propuso dividir los 150 días en 4 periodos de comparación: en el primero se asignaron las hembras con 30 días de gestación o menos $(n=23)$. En el segundo, las hembras gestantes de 31 a $60(n=13)$. El tercero estuvo comprendido por las hembras de 61 a 90 días de gestación $(\mathrm{n}=10)$, y en el cuarto, aquellas con 91 días o más de preñez $(\mathrm{n}=28)$.

Manejo zootécnico de los animales. La zootecnia de los animales se realizó en sistemas de pastoreo extensivo, con predominancia de pastos Angleton (Dichantum aristatum), Colosuana (Botrichloa pertusa), Climacuna (Dichantum annulatum) y Braquipará (Brachiaria plantaguinea). Los animales recibieron agua y sal mineralizada a voluntad. El plan sanitario en estas fincas era básico, el cual solo contaba con vermifugaciones y la aplicación de vitaminas cada tres meses.

Toma y procesamiento de muestras sanguíneas. Las muestras se colectaron por medio de venopunción yugular, previa desinfección del área (surco yugular) con yodo al 10\%. La toma de muestras se efectuó en horas de la mañana con los animales en ayuno. Se utilizaron tubos BD Vacutainer ${ }^{\circ}$ con anticoagulante EDTA con capacidad de $4 \mathrm{~mL}$ según lo descrito por MEYER et al (6), estos se rotularon y embalaron en una cava de poliestireno extendido manteniendo una temperatura de $4^{\circ} \mathrm{C}$ para el almacenamiento temporal durante el transporte al Laboratorio Clínico de la Facultad de Medicina Veterinaria y Zootecnia de la Universidad de Córdoba donde se determinaron las variables del hemograma mediante un analizador hematológico veterinario ABX Micros ESV 60 (HORIBA ABX SAS, Japón).

Análisis estadístico de datos. Todos los datos arrojados por el programa hematológico sistematizado se tabularon en una hoja de cálculo de Microsoft Excel ${ }^{\circ}$. Se determinaron mediante estadística descriptiva los valores promedio y la desviación estándar para cada una de las variables estudiadas, se realizó un análisis de varianza (ANOVA; $\mathrm{p} \leq 0,05$ ) para evaluar el efecto entre periodos de gestación para las variables estudiadas y se realizó una prueba de comparación de medias de Tukey $\mathrm{p} \leq 0,05$. Por último, se hizo un análisis de regresión lineal simple para estimar las relaciones existentes entre la variable independiente "tiempo de gestación" y las variables dependientes "líneas celulares”. Se utilizó el paquete estadístico SAS 9.0 (7).

\section{RESULTADOS}

Los resultados comparativos para el leucograma, según el agrupamiento por días de gestación de los animales objeto de estudio, no mostraron diferencias $(\mathrm{p} \geq 0,05)$ a excepción de la variable monocitos, tal como se puede apreciar en la tabla 1. 
TABLA 1

Comparación de medias por agrupamiento según días de gestación para variables del leucograma de ovejas

\begin{tabular}{|c|c|c|c|c|}
\hline & \multicolumn{4}{|c|}{ Duración de la gestación (días) } \\
\hline Variable & oa 30 & 31 a 60 & 61 a 90 & $>90$ \\
\hline Leucocitos (x103/mm3) & $8,996^{\mathrm{A}}$ & $8,700^{\mathrm{A}}$ & $10,180^{\mathrm{A}}$ & $9,464^{\mathrm{A}}$ \\
\hline Linfocitos $(\%)$ & $44,570^{\mathrm{A}}$ & $45,592^{\mathrm{A}}$ & $46,920^{\mathrm{A}}$ & $45,857^{\mathrm{A}}$ \\
\hline Monocitos (\%) & $7,339^{A}$ & $6,930^{A B}$ & $6,330^{\mathrm{AB}}$ & $6,264^{\mathrm{B}}$ \\
\hline Granulocitos (\%) & $48,091^{\mathrm{A}}$ & $47,477^{\mathrm{A}}$ & $46,750^{\mathrm{A}}$ & $47,521^{A}$ \\
\hline Eosinófilos (\%) & $4,596^{\mathrm{A}}$ & $4,369^{\mathrm{A}}$ & $5,060^{\mathrm{A}}$ & $4,557^{\mathrm{A}}$ \\
\hline Linfocitos $\left(\times 10^{3} / \mathrm{mm}^{3}\right)$ & $3,891^{\mathrm{A}}$ & $4,061^{\mathrm{A}}$ & $4,580^{\mathrm{A}}$ & $4,400^{\mathrm{A}}$ \\
\hline Monocitos $\left(\times 10^{3} / \mathrm{mm}^{3}\right)$ & $0,613^{A}$ & $0,546^{\mathrm{A}}$ & $0,580^{\mathrm{A}}$ & $0,528^{\mathrm{A}}$ \\
\hline Granulocitos $\left(\times 10^{3} / \mathrm{mm}^{3}\right)$ & $4,491^{A}$ & $4,276^{\mathrm{A}}$ & $5,020^{\mathrm{A}}$ & $4,535^{A}$ \\
\hline Eosinófilos $\left(\times 10^{3} / \mathrm{mm}^{3}\right)$ & $0,466^{\mathrm{A}}$ & $0,406^{\mathrm{A}}$ & $0,519^{A}$ & $0,442^{\mathrm{A}}$ \\
\hline
\end{tabular}

Medias en una misma fila con distinta literal son diferentes $(\mathrm{p} \leq 0,05)$

A continuación, en la figura 1 se muestra la tendencia observada de los monocitos durante la gestación en ovejas de pelo criollas.

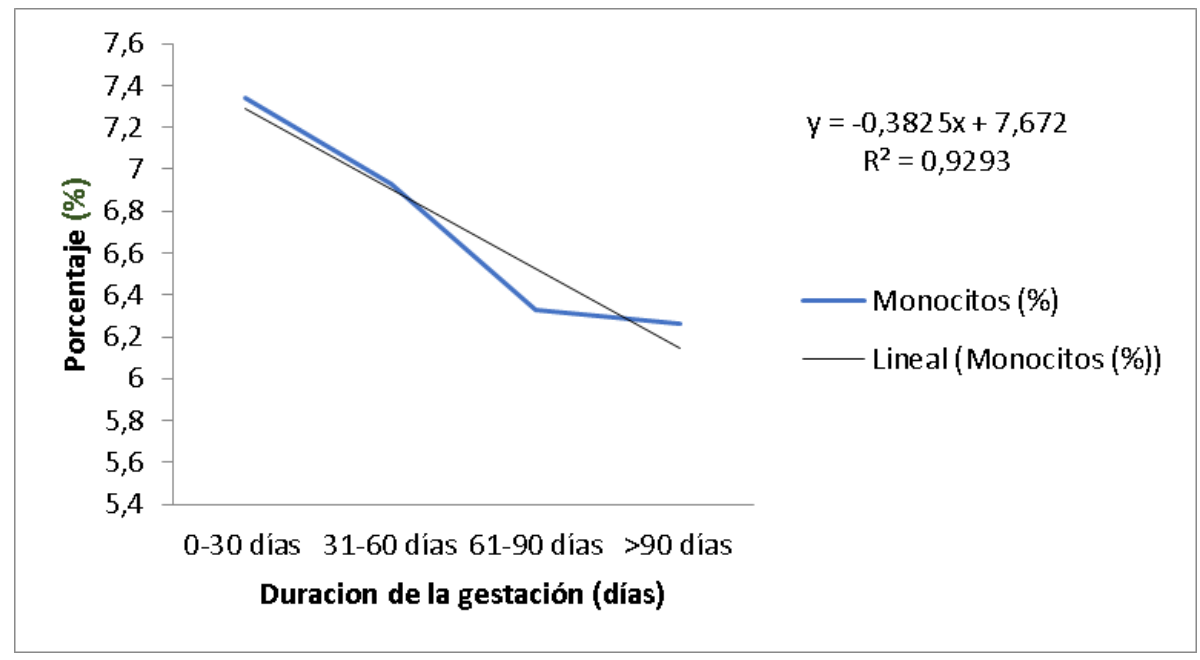

FIGURA 1

Regresión de las variables porcentaje de monocitos y tiempo de gestación

Los resultados de las variables del eritrograma y plaquetograma según los días de gestación se aprecian en la tabla 2. No encontrándose diferencia significativa $(\mathrm{p} \leq 0,05)$ en ninguna de ellas. 
TABLA 2

Comparación de medias por agrupamiento según días de gestación para variables del eritrograma y plaquetograma

\begin{tabular}{|c|c|c|c|c|}
\hline \multirow[b]{2}{*}{ Variable } & \multicolumn{4}{|c|}{ Duración de la gestación (días) } \\
\hline & o a 30 & 31 a 60 & 61 a 90 & $>90$ \\
\hline Eritrocitos $\left(\times 10^{6} / \mathrm{mm}^{3}\right)$ & $8,574^{\mathrm{A}}$ & $8,753^{A}$ & $9,329^{\mathrm{A}}$ & $8,864^{\mathrm{A}}$ \\
\hline Hemoglobina (g/dL) & $9,052^{\mathrm{A}}$ & $8,938^{A}$ & $9,190^{\mathrm{A}}$ & $8,935^{\mathrm{A}}$ \\
\hline Hematocrito $(\%)$ & $31,783^{\mathrm{A}}$ & $32,200^{A}$ & $33,690^{\mathrm{A}}$ & $32,882^{A}$ \\
\hline $\mathrm{VCM}(\mathrm{fL})$ & $37,304^{\mathrm{A}}$ & $37,077^{\mathrm{A}}$ & $36,100^{\mathrm{A}}$ & $37,107^{A}$ \\
\hline $\mathrm{HCM}(\mathrm{pg})$ & $10,608^{\mathrm{A}}$ & $10,315^{\mathrm{A}}$ & $9,970^{\mathrm{A}}$ & $10,132^{A}$ \\
\hline $\mathrm{CHCM}(\mathrm{g} / \mathrm{dL})$ & $28,613^{\mathrm{A}}$ & $27,861^{\mathrm{A}}$ & $27,520^{\mathrm{A}}$ & $27,253^{A}$ \\
\hline Plaquetas $\left(\times 10^{3} / \mathrm{mm}^{3}\right)$ & $834,6^{\mathrm{A}}$ & $882,6^{\mathrm{A}}$ & $1028,4^{\mathrm{A}}$ & $873,5^{\mathrm{A}}$ \\
\hline
\end{tabular}

VCM: volumen corpuscular medio, HCM: hemoglobina corpuscular media. CHCM: concentración de hemoglobina corpuscular media

\section{DISCUSIÓN}

Los resultados del hemograma no difieren entre los periodos de gestación, excepto para los valores relativos de monocitos, donde se observó que a medida que aumenta el tiempo de gestación, el porcentaje de monocitos disminuye. Sin embargo, los valores absolutos de monocitos fueron similares durante la gestación.

La reducción de monocitos circulantes (monocitopenia) no posee ningún tipo de relevancia clínica, ya que estas células pueden estar presentes o ausentes en la sangre periférica (8) y su aumento ocurre solo en procesos de intensa fagocitosis como la necrosis de tejidos, la hemólisis intravascular o en enfermedades supurativas crónicas $(9,10)$, también en la fase post-aguda o después de la recuperación de una infección viral y en ciertos tipos de leucemia. Sin embargo, en el presente estudio, la importancia de este valor evidencia la relación entre el tiempo de gestación con el porcentaje de monocitos circulantes (Figura 1). Por lo tanto, debido a que no se ha reportado la relación existente entre el avance de la gestación en ovejas y el número de monocitos circulantes en sangre, la presente investigacion podria establecer la base para la interpretacion clínica y determinar durante la gestación en la oveja, si el porcentaje de monocitos corresponde a los valores normales.

Se han reportado valores similares con los del presente estudio para el conteo total de leucocitos. Sandoval et al (11) obtuvieron $9,30 \times 10^{3} / \mathrm{mm}^{3}$ en ovejas infectadas por estróngilos digestivos y $7,66 \times 10^{3} / \mathrm{mm}^{3}$ en ovejas tratadas con ivermectinas, mientras que Partida et al (12) refirieron $7,92 \times 10^{3} / \mathrm{mm}^{3}$ en ovino criollo mexicano, y Couto (13) indicó $9,34 \times 10^{3} / \mathrm{mm}^{3}$ de leucocitos en ovinos criollo en Brasil.

Los valores para eosinófilos obtenidos en este estudio, superan los hallados por Medina y Callacná (3) de $0,182 \times 10^{3} / \mathrm{mm}^{3}$ en cabras gestantes. Esto podría indicar que dichas células están presentes para contrarrestar los efectos de alguna inflamación. La eosinofilia o aumento de eosinófilos se da también cuando hay descomposición de proteínas orgánicas, lo cual indicaría una función antitóxica (14). La cantidad de eosinófilos también aumenta en situaciones alérgicas, shock anafiláctico y ciertos tipos de parasitismo, en particular en los que existen estadios larvarios migratorios (15). El estado de eosinofilia observado en el presente estudio, posiblemente resultó por alguna afección parasitaria presente en los animales durante la fase experimental, debido a la resistencia que presentan a los medicamentos antiparasitarios (16). 
El número de monocitos hallados en el presente estudio, superan los $0,071 \times 10^{3} / \mathrm{mm}^{3}$ obtenidos en cabras criollas gestantes reportados por Medina y Callacná (3); igualmente, difieren con los $0,214 \times 10^{3} / \mathrm{mm}^{3}$ encontrado por Couto (13) en ovinos criollos de la raza Serrana. En general, los altos valores de los eosinófilos y monocitos se podrían explicar por la constante exposición de los animales a situaciones de permanentes lesiones o patologías de carácter inflamatorio e infeccioso que originan un aumento de estas células (17).

Los valores del eritrograma obtenidos en la presente investigación, concuerdan con valores publicados por Oliveira et al (18) en cabras brasileñas gestantes (Hematocrito 33,95 $\pm 5,93 \%$, Hemoglobina 10,94 $\pm 1,91$ $\mathrm{g} / \mathrm{dL}, \mathrm{CHCM} 32,24 \pm 0,03 \mathrm{~g} / \mathrm{dL}$ ), pero difieren con lo reportado por Medina y Callacná (14) en cabras gestantes $\left(13,4 \times 106 / \mathrm{mm}^{3}\right)$ y con los hallazgos de Grilli et al (19) en cabras gestantes $\left(13,5 \times 106 / \mathrm{mm}^{3}\right)$, lactantes $\left(14,7 \times 106 / \mathrm{mm}^{3}\right)$ y vacías $\left(15,9 \times 106 / \mathrm{mm}^{3}\right)$. Por el contrario, Avellanet et al (20) reportaron valores inferiores a los del presente estudio en ovinos de la raza Xisqueta $\left(8,30 \times 106 / \mathrm{mm}^{3}\right)$. Se sabe que la altitud influye en la cantidad de glóbulos rojos en sangre (21), lo cual se observó en un estudio realizado en Argentina con cabras gestantes de biotipo criollo $\left(16,27 \pm 2,27 \times 106 / \mathrm{mm}^{3}\right)(19)$ donde los resultados son superiores a los encontrados en el presente estudio.

Los resultados de hematocrito en el presente estudio, son superiores al 25 $\pm 4,8 \%$ reportado por Zárate et al (22) en ovejas Texel en último tercio de la gestación evaluadas en Paraguay. Sin embargo, son menores a los reportados por Partida et al (12) (35.78\%), en ovejas criollas vacías bajo un sistema de estabulación. Esta disminución del hematocrito, podría deberse a que en las ovejas en gestación se presenta una anemia por hemodilución denominada "anemia fisiológica de la gestación" (19). La hemodilución resultante del incremento en el volumen plasmático, puede tener una importancia fisiológica, debido a la reducción en la viscosidad de la sangre, de ese modo, se incrementaría el flujo de sangre a los pequeños vasos sanguíneos. La hemodilución puede mejorar el flujo sanguíneo a través de los vasos de la placenta, especialmente, en la preñez tardía para incrementar la difusión de nutrientes y oxígeno al feto (19). Cabe resaltar que, los animales muestreados en el presente estudio no mostraron esa particularidad; pues los valores de hematocrito, glóbulos rojos y hemoglobina no disminuyeron en función del avance de la gestación. Estos hallazgos podrían deberse a mecanismos compensatorios de adaptación específicos de las razas criollas manejadas en el departamento de Córdoba, Colombia.

En comparación con los valores del presente estudio, Pedreira et al (23) reportaron valores superiores de hematocrito en ovejas Merino (40,37\%) y Romney Marsh (46,90\%), e inferiores en ovejas Frisona (30,25\%), todas ellas sometidas a pastoreo continuo y bajo distintos sistemas productivos. Medina y Callacná (3) en un estudio realizado en cabras gestantes y no gestantes, reportan que existe una diferencia entre el hematocrito de ovejas gestantes $(24,1 \%)$ y no gestantes $(28,7 \%)$, lo cual estaría explicado en la anemia fisiológica durante la gestación. En Colombia se han reportado valores para hematocrito en ovejas Criollas gestantes de 31,24\% (24), lo cual coincide con lo reportado en el presente estudio e indica que corresponde a los valores normales para la especie.

Las variables de $\mathrm{Hb}, \mathrm{HCM}$ y $\mathrm{CHCM}$ que presentaron las ovejas en todos los grupos evaluados son inferiores a los reportados para ovinos (12), lo cual indica que posiblemente en las ovejas de Pelo Criollas existe un déficit en la síntesis de hemoglobina, debido posiblemente a grandes pérdidas de hierro sérico a causa de infestaciones por parásitos gastrointestinales $(15,17)$.

Avellanet et al (20), reportó un valor de plaquetas de 660 milllones/ $\mu \mathrm{L}$ en ovejas de la raza Xisqueta, el cual es inferior al encontrado en el presente estudio. Cabe resaltar, que esta diferencia pudo deberse al manejo de los animales durante el muestreo sanguíneo. Es decir, que los animales mostraron en el trombograma una trombocitosis transitoria, producto de la actividad física, la cual estimula la secreción de epinefrina y produce una esplenocontracción y por ende, una movilización de las plaquetas almacenadas hacia la circulación (25).

El hallazgo de recuentos plaquetarios altos es secundario a la hiperproducción de trombopoyetina. Esta trombocitosis secundaria puede ocurrir luego de un proceso hemorrágico o en asociación con ella, 
especialmente cuando la hemorragia causa anemia por deficiencia de hierro (25). Es necesario recalcar que los animales que habitan en el trópico bajo están más predispuestos al contacto con parásitos, entre ellos los parásitos gastrointestinales, que afectan en gran medida a los pequeños rumiantes (16), pudiendo causar, en la mayoría de los casos, pérdidas sustanciales de hierro sérico e incluso anemias crónicas, lo que provocaría la trombocitosis secundaria (26).

En conclusión, se da a conocer el primer reporte sobre el comportamiento promedio del hemograma completo de las ovejas Criollas de Pelo colombianas en etapa de gestación, en el departamento de Córdoba, Colombia. Los valores de las variables hematológicas de las ovejas Criollas de Pelo colombianas, concuerdan con los reportados por otros investigadores en razas criollas de ovejas y cabras de otros países. La presente investigación podría establecer la base para la interpretación clínica y determinar durante la gestación en la oveja, si el porcentaje de monocitos corresponde a los valores normales.

\section{ReFERENCIAS}

1. De Holcim Colombia, Fundación Social. "Guía Práctica para pequeños productores Ovinos". Colombia: ASOPROVINOS; 2011. https://studylib.es/doc/4617306/gu\%C3\%ADa-pr\%C3\%A1ctica-parapeque $\% \mathrm{C} 3 \% \mathrm{~B} 1$ os-productores-ovinos

2. Galeano L, Gómez MJ, Mesa JEG. Caracterización de los sistemas de pastoreo de pequeños rumiantes en el sur del Tolima. Revista Colombiana de Ciencia Animal. 2015; 6(1):75-85. http://revistas.ut.edu.co/index.php/ ciencianimal/article/viewFile/436/374

3. Medina LEG, Custodio MÁC. Hematological values of creole goats in two reproductive physiological states. Scientia Agropecuaria. 2014; 4(4):285-92. DOI: http://dx.doi.org/10.17268/sci.agropecu.2013.04.02

4. Barrios C. Guía práctica de ovinocultura enfocada hacia la producción de carne. Bogotá, Colombia: Bacom LTDA; 2007. https://docplayer.es/2860879-Guia-practica-de-ovinocultura.html

5. Cassab A, Morales V, Mattar S. Factores climáticos y casos de Dengue en Montería, Colombia. Rev Salud Pública. 2010; 13(1):115-128. https://doi.org/10.1590/S0124-00642011000100010

6. Meyer DJ, Coles EH, Rich LJ. Medicina de laboratório veterinária: interpretação e diagnóstico, São Paulo: Roca; 1995.

7. Statistical Analysis System Institute (SAS). SAS/STAT (Relase 9.0). USA: Cary, NC; 2001.

8. Roubies N, Panousis N, Fytianou A, Katsoulos PD, Giadinis N, Karatzias H. Effects of age and reproductive stage on certain serum biochemical parameters of Chios sheep under Greek rearing conditions. J Vet Med A Physiol Pathol Clin Med. 2006; 53:277-281. https://doi.org/10.1111/j.1439-0442.2006.00832.x PMid:16901268

9. Kraft WDU. Diagnóstico de laboratorio clínico en veterinaria. Barcelona: EDIMSA; 2000.

10. Burkhard MJ. Causas y efectos de interferencia con mediciones y exámenes de laboratorio clínico. In Kirk RW BJ. Terapéutica Veterinaria en peque-os animales. Barcelona: McGraw-Hill Interamericana; 1995.

11. Sandoval E, Morales G, Pino L, Jiménez D, Márquez O. Evaluación del comportamiento en ovejas a pastoreo como criterio para determinar la susceptibilidad a la infección con estrongilos digestivos. REDVET. 2007; 8(9):090907. URL Disponible en: www.veterinaria.org/revistas/redvet/n090907.html

12. Partida S, Uribe L, Butrón A. Contribución Al Estudio De Parámetros Hemáticos En Ovinos Criollos Bajo Las Condiciones De La Granja Experimental, Chapingo. Chapingo México: Universidad Autónoma Chapingo. Departamento de Ense-anza e Investigación en Zootecnia; 2011. https://zootecnia.chapingo.mx/ assets/11 partida-uribe.pdf

13. Couto HAK. Caracterización genética y perfil hematológico y bioquímico en ovinos de raza "Criolla Landana Serrana" del planalto serrano catarinense-Santa Catarina, Brasil. [Tesis doctoral]. Brasil: Universidad de León, Facultad de Veterinaria; 2010. 
14. Moreno F, Builes J, Cadavid J. Evaluación de 30 parámetros hemáticos en bovinos Bos indicus en los municipios de San Juan de Urabá y Arboletes del Uraba Antioque-o. Colombia: Universidad CES; 2008. URL Disponible en: http://bdigital.ces.edu.co:8080/repositorio/bitstream/10946/550/1/Evaluacion_parametros_hemaliticos.pdf

15. Reece W. Fisiología de los animales domésticos. $12^{\circ}$ ed. Editorial Zaragoza, Espa-a: Acribia S.A; 2004

16. Herrera Y, Vergara J, Ensuncho C, Causil L. Frecuencia de parásitos gastrointestinales en burros criollos (Equus africanus asinus) en el departamento de Córdoba, Colombia. Rev Colombiana Cienc Anim. 2016; 8(2):159-166. https://doi.org/10.24188/recia.v8.n2.2016.183

17. Juárez LM. Revisión Farmacológica [Online]. México: UNAM; 2016 [consultado 2017 Octubre 12]. URL Disponible en: http://www.facmed.unam.mx/deptos/familiar/atfm123/revfarma.html

18. Oliveira MGC, Nunes TL, Paiva ALC, Bezerra TCG, Fernandes NS, Vale AM, et al. Aspectos hematológicos de caprinos (Capra hircus) da raça Canindé criados no Rio Grande do Norte. Pesquisa Veterinária Brasileir. 2012; 32(Supl 1):4-8. https://doi.org/10.1590/S0100-736X2012001300002

19. Grilli D, Paez S, Candela M, Egea V, Sbriglio L, Allegretti L. Valores hematológicos en diferentes estados fisiológicos de cabras biotipo criollo del Ne De Mendoza, Argentina. Vo Congreso de Especialistas en Pequeños Rumiantes y Camélidos Sudamericanos, Mendoza, Argentina. Universidad Juan Agustín Maza. 2007. URL Disponible en: http://www.produccion-animal.com.ar/produccion_caprina/produccion_caprina/79-grilli.pdf

20. Avellanet R, Cuenca R, Pastor J, Jordana J. Parámetros hematológicos y bioquímico clínicos en la raza ovina Xisqueta. Arch Zootec. 2007; 56 (Sup 1):497-501. http://www.uco.es/organiza/servicios/publica/az/php/ img/web/01_08_38_21ParametrosAvellanet.pdf

21. Beall CM, Decker MJ, Brittenham GM, KUshner I, Gebremedhin A, Strohl KP. An Ethiopian pattern of human adaptation to hihg-altitude hypoxia. Procs Natl Acad Sci USA 2002; 99(26): 17215-17218. DOI: https:// doi.org/10.1073/pnas.252649199 PMC:139295

22. Zárate R, Pedrozo R, Acosta R, Lara M, Báez M, González A. Perfiles metabólicos en ovejas texel en los periodos de preservicio, último tercio de gestación e inicio de lactancia. Compend Cienc Vet. 2014; 4(2):39-46. URL Disponible en: http://scielo.iics.una.py/pdf/ccv/v4n2/v4n2a07.pdf

23. Pedreira KM, Schuh A, Fernández C, Decaminada E, Coppola M, Miralles M, et al. Perfiles hematológicos de ovinos bajo distintos sistemas productivos en Argentina. Argentina: FCV- UBA; 2007. http://www.fvet.uba.ar/ archivos/hospital/Perfiles-hematologicos-de-ovinos-bajo-distintos-sistemas-pro.pdf

24. Maza L, Cardona J, Vergara O. Análisis del perfil metabólico de hembras Ovinas criollas gestantes en condiciones De pastoreo extensivo. Universidad del Zulia. Venezuela. Revista Científica, FCV-LUZ. 2011; 21(4):335-339. http://produccioncientificaluz.org/index.php/cientifica/article/view/15657

25. Zabala N, Hannaoui E. Comparación del contaje plaquetario empleando diferentes metodologías, en pacientes con púrpura trombocitopénica y síndromes mielodisplásicos. Saber, Universidad de Oriente, Venezuela. 2013; 25(3):259-264. https://www.redalyc.org/articulo.oa?id=427739463004

26. Aguiló J. Valores hematicos. Clin Vet Pequeños Anim. 2001; 21(2):75-85. https://ddd.uab.cat/pub/ clivetpeqani/11307064v21n2/11307064v21n2p75.pdf

Los autores permiten a RECIA reimprimir el material publicado en él. En caso de que un autor quiera traducir o usar una publicación parcial o completa de nuestro Diario, el autor debe obtener un permiso por escrito del editor de la revista.

CC BY 